Document downloaded from:

http://hdl.handle.net/10251/137419

This paper must be cited as:

Baeza González, LM.; Thompson, DJ.; Squicciarini, G.; Denia, FD. (2018). Method for obtaining the wheel-rail contact location and its application to the normal problem calculation through CONTACT. Vehicle System Dynamics. 56(11):1734-1746.

https://doi.org/10.1080/00423114.2018.1439178

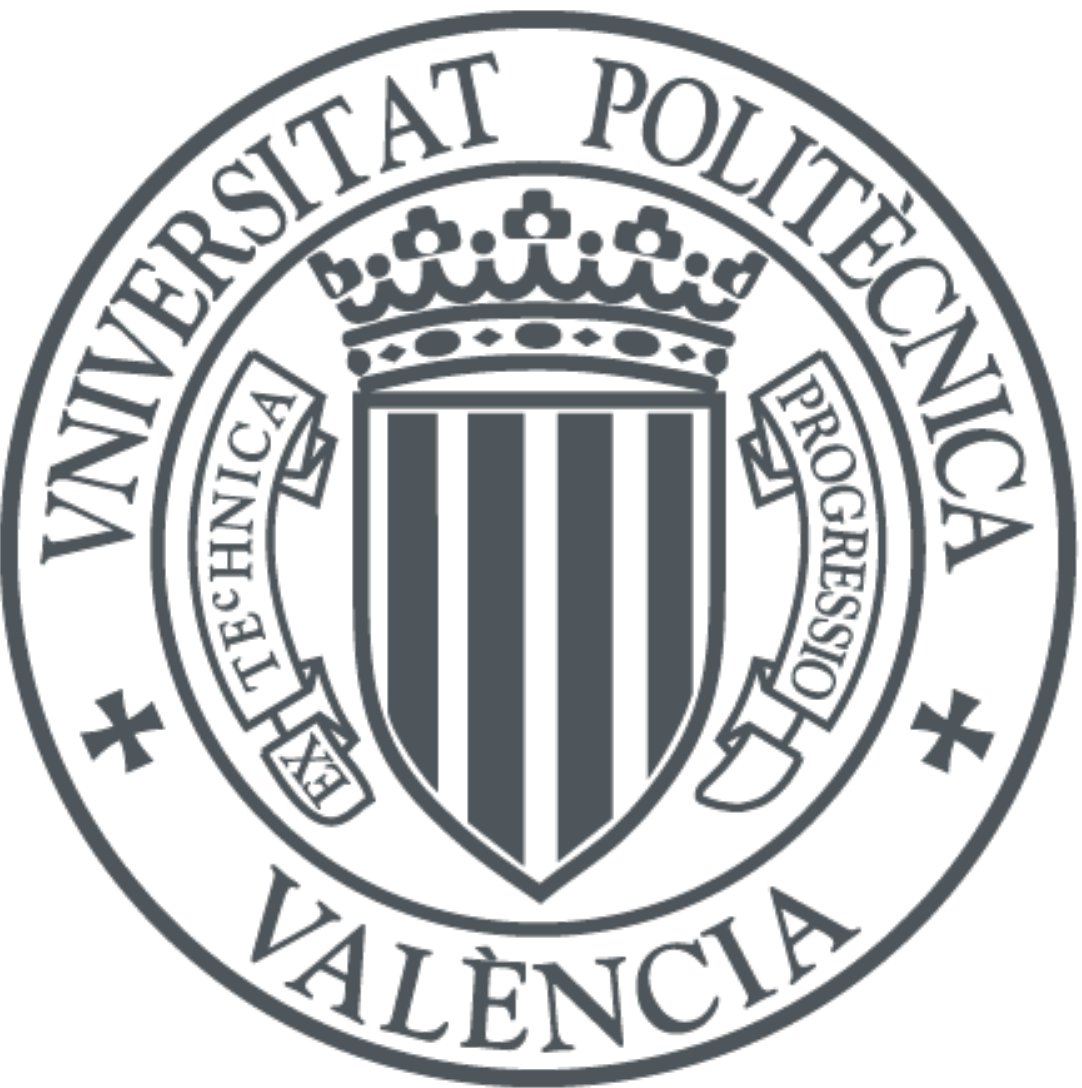

The final publication is available at

https://doi.org/10.1080/00423114.2018.1439178

Copyright Taylor \& Francis

Additional Information 
DOl: 10.1080/00423114.2018.1439178

\title{
METHOD FOR OBTAINING THE WHEEL-RAIL CONTACT LOCATION AND ITS APPLICATION TO THE NORMAL PROBLEM CALCULATION THROUGH 'CONTACT'
}

\author{
Luis Baeza $^{1 *}$, David J. Thompson ${ }^{1}$, Giacomo Squicciarini ${ }^{1}$, Francisco D. Denia ${ }^{2}$ \\ ${ }^{1}$ Institute of Sound and Vibration Research, University of Southampton, Southampton SO17 1BJ, UK. \\ ${ }^{2}$ Centro de Investigación en Ingeniería Mecánica, Universitat Politècnica de València, Camino de Vera s/n, \\ 46022 Valencia, Spain. \\ *E-mail: L.Baeza@isvr.soton.ac.uk
}




\title{
METHOD FOR OBTAINING THE WHEEL-RAIL CONTACT LOCATION AND ITS APPLICATION TO THE NORMAL PROBLEM CALCULATION THROUGH 'CONTACT'
}

\author{
Luis Baeza $^{1 *}$, David J. Thompson ${ }^{1}$, Giacomo Squicciarini ${ }^{1}$, Francisco D. Denia ${ }^{2}$ \\ ${ }^{1}$ Institute of Sound and Vibration Research, University of Southampton, Southampton SO17 1BJ, UK. \\ ${ }^{2}$ Centro de Investigación en Ingeniería Mecánica, Universitat Politècnica de València, Camino de Vera s/n, \\ 46022 Valencia, Spain. \\ * E-mail: L.Baeza@isvr.soton.ac.uk
}

\begin{abstract}
This work presents a robust methodology for calculating inter-penetration areas between railway wheel and rail surfaces, the profiles of which are defined by a series of points. The method allows general three-dimensional displacements of the wheelset to be considered, and its characteristics make it especially suitable for dynamic simulations where the wheel-rail contact is assumed to be flexible. The technique is based on the discretization of the geometries of the surfaces in contact, considering the wheel as a set of truncated cones and the rail as points. By means of this approach, it is possible to reduce the problem to the calculation of the intersections between cones and lines, the solution for which has a closed-form expression. The method has been used in conjunction with the CONTACT algorithm in order to solve the static normal contact problem when the lateral displacement of the wheelset, its yaw angle and the vertical force applied in the wheelset centroid are prescribed. The results consist of smooth functions when the dependent coordinates are represented as a function of the independent ones, lacking the jump discontinuities that are present when a rigid contact model is adopted. Example results are shown and assessed for the normal contact problem for different lateral and yaw positions of the wheelset on the track.
\end{abstract}

KEYWORDS: Flexible contact, CONTACT, Variational theory, Railway dynamics, Simulation.

To be published in Vehicle System Dynamics.

Date of submission 11-Oct-2017

Date of acceptance 11-Jan-2018

https://doi.org/10.1080/00423114.2018.1439178 


\section{INTRODUCTION}

In railway dynamics, to determine the wheel-rail contact position, the contact is often considered as rigid, which allows it to be modelled through kinematic constraints that reduce the number of degrees of freedom of the railway wheelset on the rigid track to four. This approach can provide the simplest formulations of the wheelset equation of motion whenever simplified wheel and rail profile geometries are adopted: wheel profiles approximated by cones and rail profiles by knife-edges [1]; or wheel and rail profiles approximated by the corresponding osculating circles [2], which are the circles passing through the contact point and a pair of additional points on each profile that are infinitesimally close to the contact point. However, the computation of the kinematic constraints for the real wheel and rail profiles requires a numerical procedure, the implementation of which can be complex [3]. The fact that there may be several contact points for a single wheel-rail pair may make it impossible to find a satisfactory solution of the contact location problem. For some wheelset positions, the initial guess for the contact parameters has to be so close to the right solution that the Newton-Raphson scheme is ineffective. Furthermore, frequently, invalid positions may be obtained that imply inter-penetration between the surfaces. These problems can be mitigated by changing the wheelset independent variables [4], or using suitable interpolation functions [5]. These and other issues associated with the rigid contact model are solved through methodologies based on a penalty function that model the contact as elastic.

At present, many of the commercial dynamic simulation packages, as well as models that simulate the dynamic interaction of a railway vehicle with the track, are based on the flexible contact hypothesis. The methodology behind this technique is to adopt a penalty force at points where penetration between the undeformed surfaces of the wheel and the rail takes place [6]. The force is determined according to a contact mechanics model and, therefore, the procedure for calculating the inter-penetration areas of the undeformed geometries must be in line with the adopted contact mechanics theory. In this sense, the Hertz model is the most commonly used theory due to its simplicity and computational efficiency [7-10]. In the literature techniques are also presented based on the finite element method [11], multiHertzian techniques [12,13], as well as other approaches based on virtual penetration [14]. In references $[15,16]$ calculations have been published using the variational theory [17] for contact point positions obtained through the program Simpack [7].

One of the main difficulties of techniques based on flexible contact, is to detect efficiently the areas of penetration between wheel and rail undeformed surfaces. In references $[18,19]$ parameterization of the wheel and the rail surfaces is proposed, proceeding to the numerical calculation of a penetration vector that is orthogonal to both surfaces. An equivalent procedure is to calculate the minimum distance vector between the rail and wheel surfaces $[20,21]$. The above methods perform the calculation of contact points for a three-dimensional (3D) problem, which implies a greater degree of numerical complexity than for a two-dimensional (2D) problem in which the effect of yaw angle is neglected. A possible way to reduce the problems associated with $3 \mathrm{D}$ models is to solve a set of $2 \mathrm{D}$ problems corresponding to several vertical sections of the wheel and rail [10]. A methodology that aims to reduce the size of 3D models was presented in [22] (a detailed description of this method can be found in [23]). The method was developed for rigid contact, but has been adapted to flexible contact in [24, 25]. In this procedure, for each yaw angle of the wheelset, a three-dimensional curve is first determined that contains all the possible contact points in the wheel profile corresponding to those wheel points, the normal vectors of which are contained in a plane that is perpendicular to the track direction.

Most of the existing methods for calculating the wheel and rail inter-penetration areas (as well as the contact-point calculation techniques for rigid contact) involve associated systems of non-linear 
algebraic equations. Therefore, unsatisfactory solutions (e.g. when one of the inter-penetration areas is not detected) and numerical problems (Jacobian matrix singularity, etc.) may occur.

In the present work a methodology is proposed that aims to eliminate the problems associated with the calculation of the inter-penetration areas. The method is valid for general geometries of wheel and rail profiles. The methodology is explained in section 2 , and how the technique is adapted to normal contact calculations through the CONTACT algorithm is shown in section 3. In section 4 results are presented corresponding to static frictionless calculations, adopting the variational theory as the contact model.

\section{CONTACT POINT LOCATION}

The method proposed for estimating the inter-penetration areas between wheel and rail surfaces is based on the discretization of the wheel and rail geometries: their surfaces are considered to be represented by a set of truncated cones (for the wheel) and a set of points (for the rail), as shown in the sketch of Figure 1. The calculation of the inter-penetration areas therefore involves the computation of intersections between lines associated with points of the rail and the truncated cones of the wheel surface. The method is based on the fact that the intersection between a line and a cone has a closedform expression, even if the orientation of the cone (wheelset) is modelled by means of a sequence of rotations.

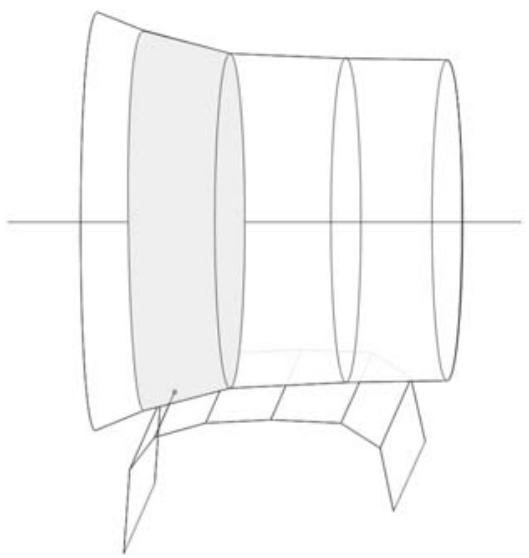

Fig. 1. Sketch of the geometric solution method. The geometries of the wheel and rail are discretised into sets of cones and generatrix lines respectively. The intersection between these surfaces is found analytically.

In order to define the contact location problem, a fixed coordinate system is considered the origin of which corresponds to the position of the wheelset centroid when it is centred on the track; the $\mathbf{X}$-axis is in the rail direction, the $\mathbf{Z}$-axis points vertically upward, and the $\mathbf{y}$-axis is obtained from the righthand rule. A coordinate system is defined for the wheelset given by two displacements $(y, z)$ which indicate the position of the wheelset centre, and a sequence of rotations around the first and third axes, with $\mathbf{A}$ being the associated rotation matrix. The $r-$ th point on the rail and the $w-$ th one on the wheel (when the wheelset is centred on the track) are defined by the coordinates $\left\{\bar{y}_{r}, \bar{z}_{r}\right\}$ and $\left\{y_{w}, z_{w}\right\}$ , respectively. The calculation of the intersection between one of the wheel cones with a straight line on the rail corresponds to the solution of the following system of equations:

$$
\left\{\begin{array}{l}
\bar{x} \\
\bar{y}_{r} \\
\bar{z}_{r}
\end{array}\right\}=\left\{\begin{array}{l}
0 \\
y \\
z
\end{array}\right\}+\mathbf{A}\left[\begin{array}{ccc}
\cos \varepsilon & 0 & -\sin \varepsilon \\
0 & 1 & 0 \\
\sin \varepsilon & 0 & \cos \varepsilon
\end{array}\right]\left\{\begin{array}{c}
0 \\
\alpha y_{w+1}+(1-\alpha) y_{w} \\
\alpha z_{w+1}+(1-\alpha) z_{w}
\end{array}\right\},
$$


in which $\bar{x}, \varepsilon$ and $\alpha$ are the unknowns of the problem; $\bar{x}$ is the longitudinal coordinate of the intersection, $\varepsilon$ is its angular position with respect to the $\mathbf{y}$-axis around the wheel circumference, and $\alpha$ is a parameter that defines the position of the intersection in the truncated cone generatrix line segment. The problem is solved by calculating the coordinates of the intersection $\left\{\begin{array}{lll}\bar{x} & \bar{y}_{r} & \bar{z}_{r}\end{array}\right\}^{\mathrm{T}}$, which must be real, and $\alpha$ must be contained in the interval ]0,1]. An analytical solution of the previous system of equations is shown in the Appendix, where a number of formulae are provided to establish when the intersection conditions are satisfied. Without loss of generality, this procedure can be adapted to other coordinate systems.

The result will be one or more inter-penetration areas. Due to the topology of the wheel and rail surfaces, each line of the rail defined by $\left\{\bar{y}_{r}, \bar{z}_{r}\right\}$ that intersects the wheel surface approximated by cones, will produce two intersections. These two points will define a line segment, which corresponds to the leading edge $\left\{\begin{array}{lll}\bar{x}_{\ell} & \bar{y}_{r} & \bar{z}_{r}\end{array}\right\}^{\mathrm{T}}$ and to the trailing edge $\left\{\begin{array}{lll}\bar{x}_{t} & \bar{y}_{r} & \bar{z}_{r}\end{array}\right\}^{\mathrm{T}}$ of the inter-penetration area, as shown in Figure 2. The corresponding mid-point of the largest segment is identified as the nominal contact point.

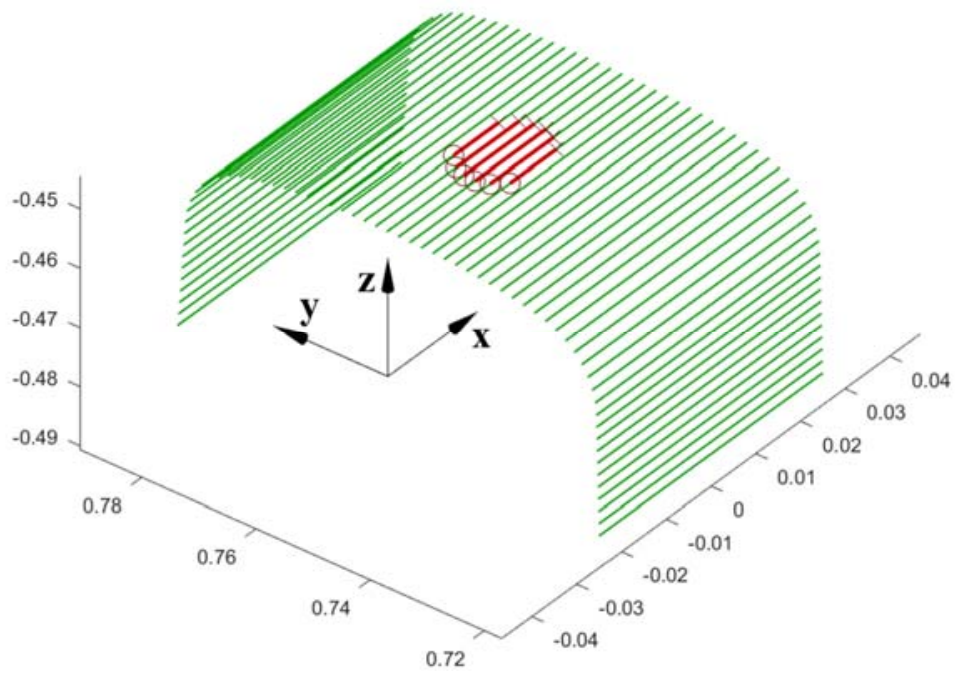

Fig. 2. The figure shows an example of the inter-penetration area on the rail. The lines that define the rail are plotted in thin green lines; the segments that result from the inter-penetration calculation are plotted in bold red lines. Marker o denotes the trailing points of the inter-penetration area, whereas the marker $\mathrm{x}$ denotes the leading points. Dimensions in $m$.

The intersections can be associated with one cone of the wheel if Equation (1) produces two points that satisfy the intersection conditions (see the intersection conditions in the Appendix); it is also possible that a line of the rail $\left\{\bar{y}_{r}, \bar{z}_{r}\right\}$ intersects two different cones and, in such case, two cones produce a solution that satisfies the intersection conditions; this case is only possible if the yaw angle $\psi$ is not zero.

Through this approach, the problem is limited to determining which rail lines intersect with which cones of the wheel. Although it is possible to check all the possible line-cone combinations, there are alternative promising ways to reduce the cost associated with the calculation of the inter-penetration areas; examples are:

1. Making pre-calculations with very few points of the profiles, the positions of which are modified to new positions away from the solids (see Figure 3). In this way, the approximate position of the inter-penetration areas can be estimated more quickly. 
2. Setting the points of the wheel such that the slope increment between two consecutive points is constant. This criterion would only require a greater number of points where the curvature of the wheel profile is large, whereas only two points are required if the section is conical.

3. Establishing strategies that allow impossible combinations to be neglected according to the topology of the profiles. For example, if the $r-1$ point of the rail does not intersect with cone $w$ and the $r$-th one does intersect with it, there is no need to check rail lines with index smaller than $r$ with cones with index larger than $w$.

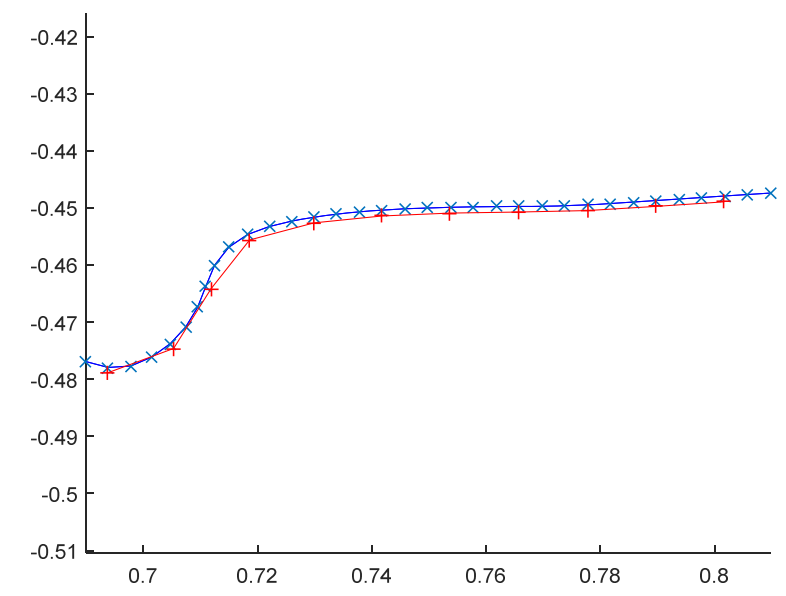

Fig. 3. A first estimation of the areas of inter-penetration is carried out through a rough definition of the profiles (marker + in the figure for the wheel profile) in which the original position of the points has been modified, moving them to new positions outside the solid surfaces. Based in this preliminary estimation, the calculation of the inter-penetration areas for the points in the actual position (marker $\mathrm{x}$ ) is then performed.

Dimensions in $m$.

\section{NORMAL CONTACT PROBLEM}

In this work, the variational theory from Kalker [17] is adopted for the normal contact model. The calculation by means of this contact theory requires, firstly, to define the contact plane. This is the plane that is tangent to the wheel (or rail) surface at the nominal contact point. Any parameter (normal traction, displacement,...) associated with the contact is defined at the projected position on the contact plane. The potential contact area is introduced as a regular area (normally rectangular) within the contact plane containing at least all wheel-rail contact points. A rectangle of the contact plane containing the interpenetration area fulfils the potential contact area condition because the actual contact area is smaller than the inter-penetration area. The procedure in CONTACT [17] is based on the discretization of the potential contact area in a regular mesh on the elements of which it is assumed that the normal traction is constant. By assuming a half-space elastic model, this hypothesis allows closed-form expressions to be established for the elastic influence coefficients that, in the constitutive equation, relate the displacements in the centre of the elements with the normal tractions (the influence coefficients can be found in Ref. [26]).

The variational theory requires the calculation of the distance between the undeformed surfaces of the wheel and the rail. To achieve this, at each point of inter-penetration given by the coordinates of the rail $\left\{\bar{x}_{r}, \bar{y}_{r}\right\}$ it is considered that the geometry of the wheel corresponds to the cone for which the intersection has occurred. If the intersection of one rail line occurs for two different cones (which can only occur for non-zero yaw angles), the geometric properties of the two cones involved are averaged. Thus, for each intersection line segment, the distance between the undeformed surfaces along the segment is estimated by means of a circular arc passing through the end points of the line segment; the radius of the arc is the actual wheel radius at the wheel cone divided by the cosine of the conicity angle. 
The undeformed distances for the elements of the potential contact area are then calculated by interpolation between the distances obtained for each segment.

According to the CONTACT algorithm, the calculation of the contact traction distribution is obtained through the equation:

$$
2 \mathbf{D} \mathbf{p}=\mathbf{h},
$$

where $\mathbf{p}$ is the normal traction distribution in the elements located in the contact area, $\mathbf{h}$ contains the distance between the rail and wheel undeformed surfaces, and $\mathbf{D}$ is the matrix of elastic coefficients. The procedure requires an assumption about which elements of the potential contact area belong to the contact area. For this, it is initially considered that all elements with a distance $h$ that is positive belong to the contact area. Next, a solution will be obtained through Equation (2), from which the elements of the contact area will be redefined as those with positive normal traction. The calculation will be performed again iteratively until the normal tractions of all the elements are positive. The CONTACT algorithm [17] proposes to check if any element has been wrongly removed from the contact area (which happens if $2 \mathbf{D p}-\mathbf{h}$ produces any negative component when all the elements of the initial potential contact are included), but in our experience this case has never been detected.

As a result of the normal contact calculation, the contact area and the normal traction distribution are obtained. From this it is possible to determine the resultant force associated with the normal traction distribution, which will be considered to be applied at the corresponding centre of pressure (the point at which the total sum of the normal traction distribution acts on the solid, causing a force to act through that point).

\section{RESULTS}

The present section shows results obtained through the proposed method. The calculations have been performed for an ORE S1002 wheel profile on 60E1 (UIC60) rails. The wheel and rail profiles are sampled at $0.1 \mathrm{~mm}$ intervals, which is a finer interval (in this study, accuracy is more important than computational cost). The inclination of the rails is $1 / 40$ and the track gauge measured at $14 \mathrm{~mm}$ below the rail top plane is the UIC standard $1435 \mathrm{~mm}$. The wheel diameter is $900 \mathrm{~mm}$, which is measured at $70 \mathrm{~mm}$ from the flange back; the distance between the inside wheel surfaces is $1360 \mathrm{~mm}$. The Young's modulus and Poisson's ratio of wheel and rail materials are $2.1 \times 10^{11} \mathrm{~N} / \mathrm{m}^{2}$ and 0.3 respectively. For these calculations, the program for contact location developed in this study, despite adopting an overreduced distance between points, is found to be 10 times faster than our implementation of CONTACT.

The calculations have been carried out for a free wheelset under frictionless static conditions. In these computations the lateral displacement and the yaw angle of the wheelset are prescribed, and a vertical load $Q$ is applied in the wheelset centroid (it is chosen as $Q=200 \mathrm{kN}$ in all the calculations). As a result of applying the static equilibrium conditions, the vertical position of the centroid $Z$ and the roll angle (around $\mathbf{x}$-axis) $\phi$ are computed, as well as the lateral force $Y$ applied at the centroid and the moments with respect to the $\mathbf{y}-$ and $\mathbf{z}$-axes that balance the system. This calculation is carried out following a Newton-Raphson scheme.

Figure 4 shows the vertical displacement $Z$ and roll angle $\phi$ as a function of the lateral displacement $y$ of the wheelset. The calculations were carried out for several yaw angles $\psi$. The resulting plots are similar to those that can be found in the literature for rigid wheel/rail contact except that jump discontinuities are found in the latter [7]; the present functions are smoother than those obtained through a rigid approach. 

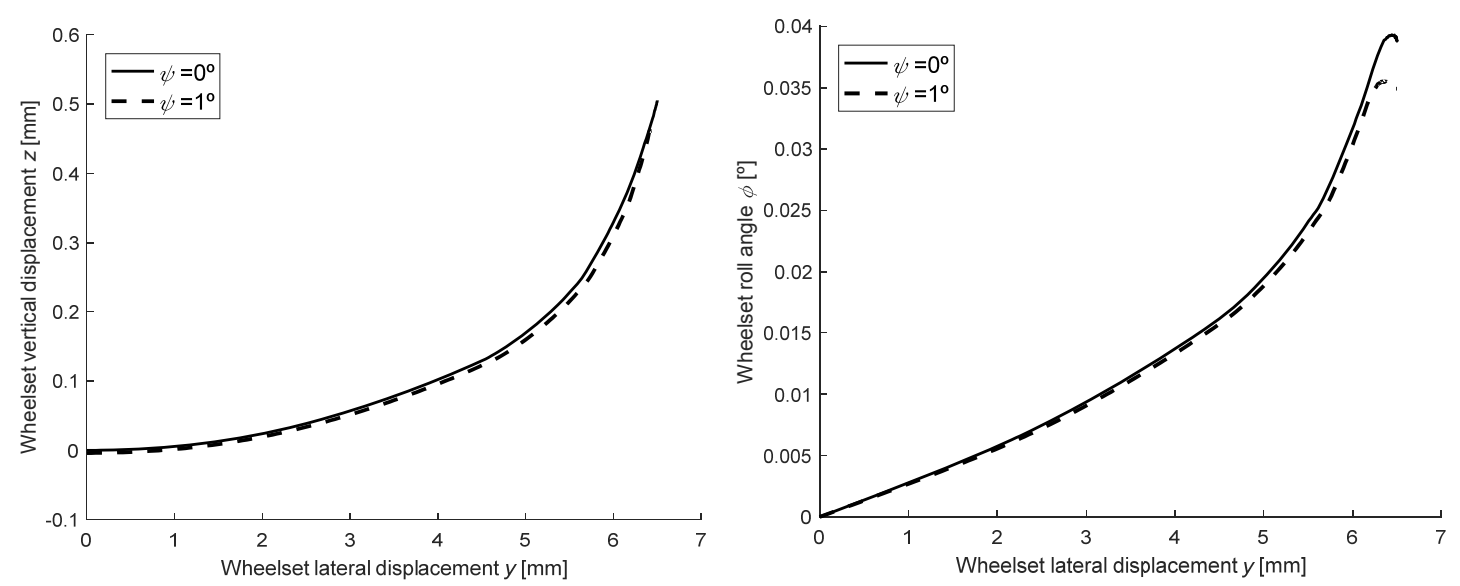

Fig. 4. Dependent coordinates (z and $\phi$ ) vs wheelset lateral displacement $y$, for two yaw angles $\psi$. The calculation is obtained through a static approach where vertical force, lateral displacement and yaw angle are prescribed.

Figure 5 plots the lateral/vertical force ratio $Y / Q$ and the moment $M_{z}$ with respect to the $\mathbf{z}$-axis that balance the static problem (the moment $M_{z}$ appears when the right and left contact points are in different $\mathbf{y z}$ planes, which is possible if $\psi \neq 0$ ). It can be seen that at a lateral displacement of $6.3 \mathrm{~mm}$ the lateral/vertical force ratio $Y / Q$ will exceed 0.8 , which is the most commonly accepted derailment limit (EN 14363 [27] establishes this value as the critical limit). These $Y / Q$ and $M_{z}$ plots also allow the gravitational stiffness to be assessed, which is the steering effect that does not depend on creep forces. The plot of $M_{z}$ shows that the moment, which has a relatively small effect, is negative before flange contact occurs, and consequently the gravitational stiffness is also negative. The moment becomes positive (stable) when there is contact at the flange due to the longitudinal shift of the contact point for large yaw angles (as will be seen below). The moment is zero for zero yaw angle.
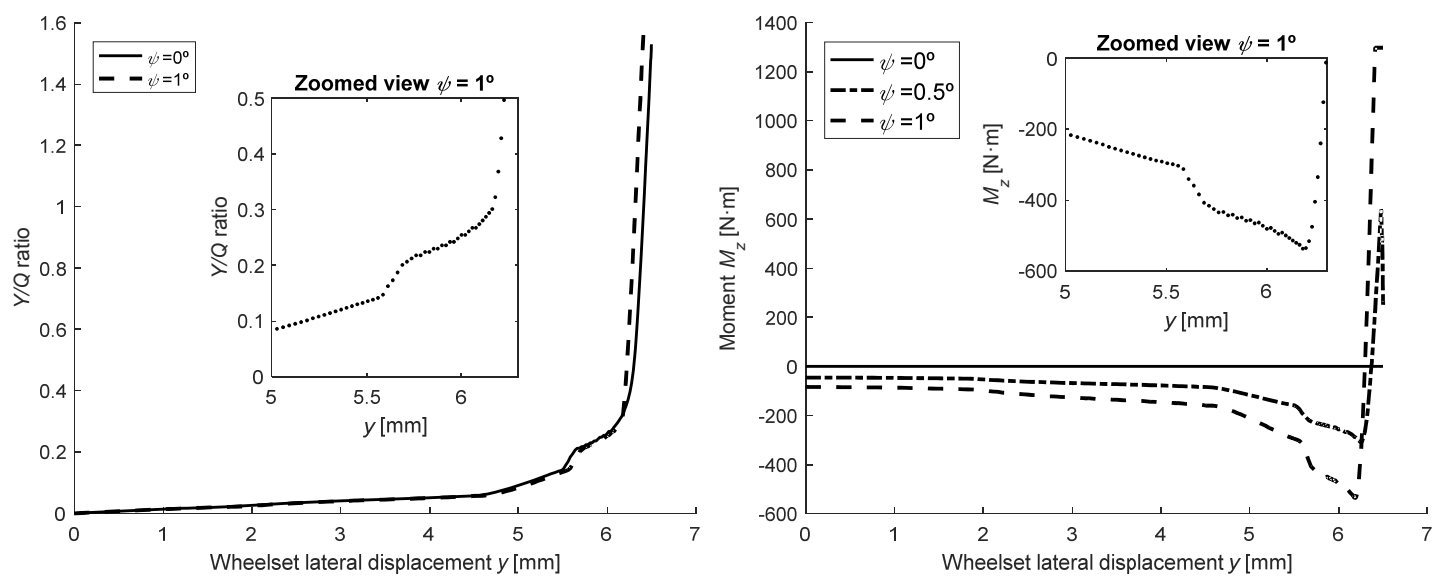

Fig. 5. Lateral/vertical force ratio and $\mathbf{z}$-axis moment that balance the static problem when lateral displacement and yaw angle are prescribed. The plots show zoom views of the discontinuity at $y=5.6 \mathrm{~mm}$; the dots correspond with the solutions that were found.

Figure 6 presents the normal traction distribution for several values of wheelset lateral displacement. In this study, results can be seen where the distribution is non-Hertzian $(y=0)$, approximately Hertzian 

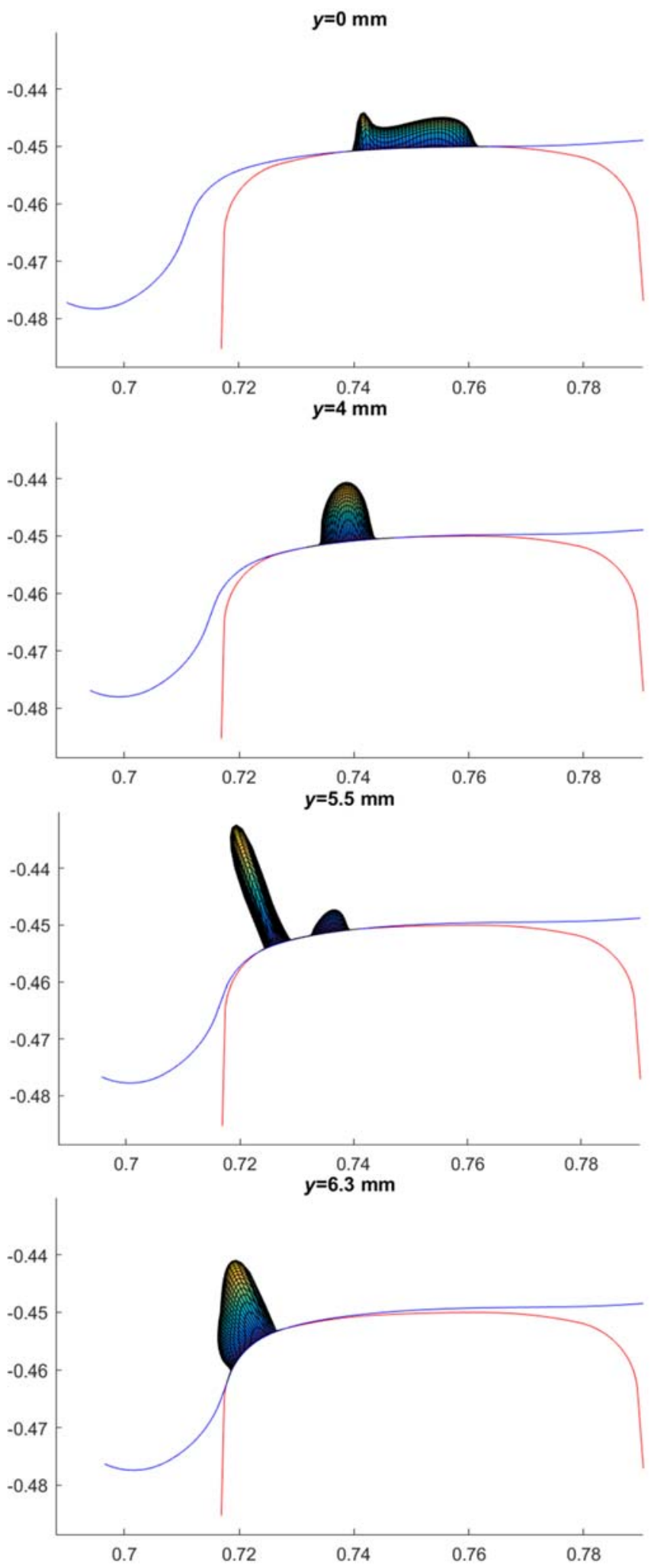

Fig. 6. Normal traction distributions for different wheelset lateral positions. The calculations were carried out for yaw angle of 0 . Dimensions in $m$. 
contact model for conformal cases could be questioned; however the $Y / Q$ ratio for this case is high and would rarely happen. For the left-side wheel/rail pair, an approximately Hertzian contact patch is found when the lateral displacement is in the following lateral ranges: [-6.4,-2.2], [3.4,4.5], [5.7,6.1] $\mathrm{mm}$. Multiple contacts are found when the lateral displacement is in the range [4.5,5.7] $\mathrm{mm}$. There is conformal contact when the lateral displacement is larger than $6.1 \mathrm{~mm}$. As a consequence of these results, Hertzian contact occurs for both wheels simultaneously for the intervals $\pm[3.4,4.5]$ and \pm $[5.7,6.1] \mathrm{mm}$.

The shape of the normal traction distribution is consistent with the distance between the undeformed surfaces $\mathbf{h}$ (which is the basis of the virtual inter-penetration contact theories [14]). This magnitude is plotted in Figure 7 for the selected displacements of the wheelset corresponding to Figure 6.
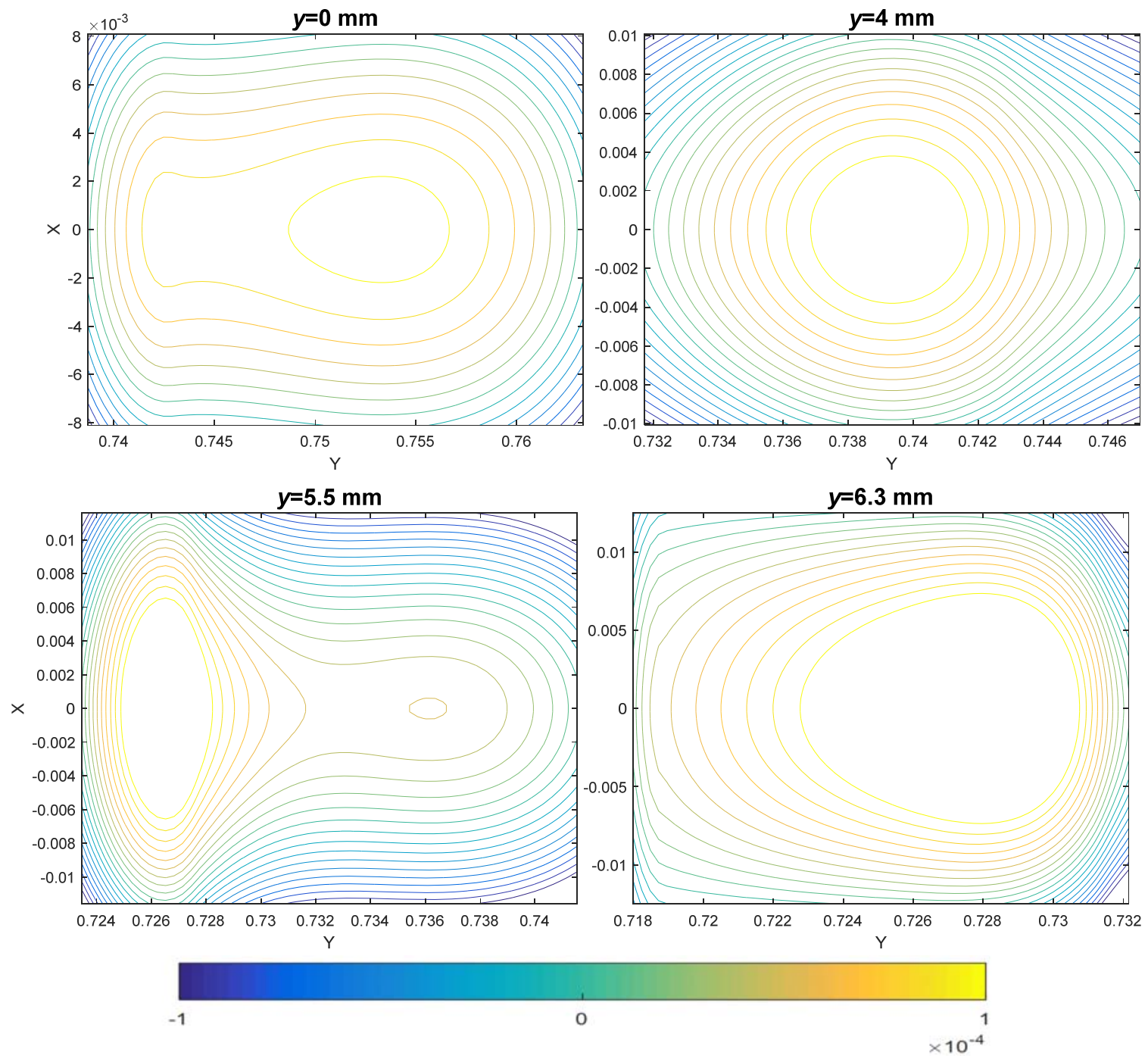

Fig. 7. Distance between wheel/rail undeformed surfaces. The calculation was made for the potential contact area that encloses the inter-penetration. Dimensions in $m$.

In Figure 8 a three-dimensional view is given of three wheel/rail contacts on the rail surface. The calculations were carried out for different wheelset positions. The normal tractions are represented for these positions. It can be seen that for the wheelset position (a) $y=6.3 \mathrm{~mm}$ and $\psi=1^{\circ}$, the contact area advances towards positive longitudinal positions due to the contact of the rail with the wheel flange. This shift can be seen if the contact traction position is compared with the ones for a centred wheelset 
(b) and a negative lateral displacement (c). This effect results in a sign change of the gravitational stiffness with respect to the yaw angle, which changes from negative for small lateral displacements of the wheelset, to positive when the flange is in contact (see Figure 5(b)).

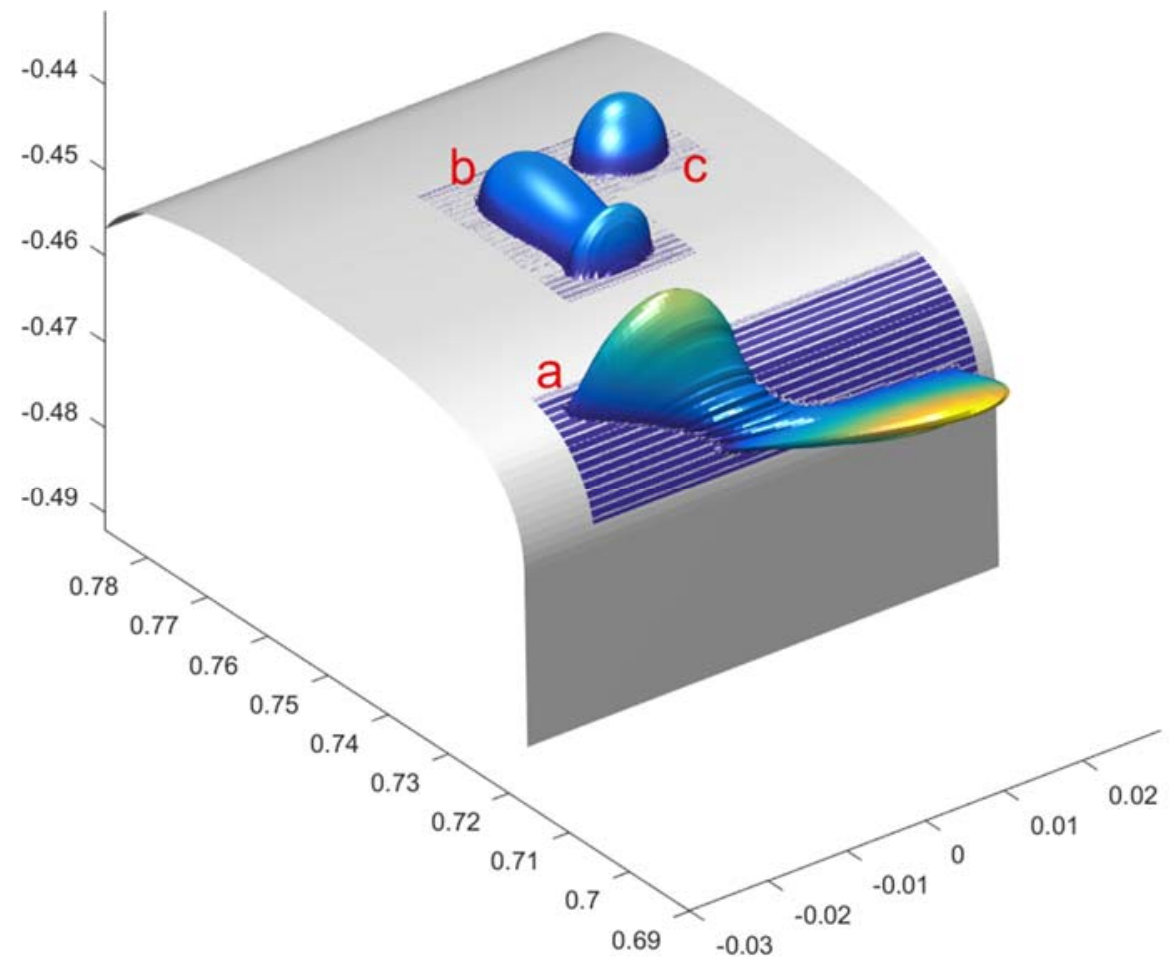

Fig. 8. Normal traction distribution on the rail surface for different wheelset positions: a) $y=6.3 \mathrm{~mm}, \psi=1^{\circ}$; b) $y=0, \psi=0$ (centred); c) $y=-6.3 \mathrm{~mm}, \psi=-1^{\circ}$. Dimensions for $\mathbf{x}, \mathbf{y}, \mathbf{z}$-axes are $\mathrm{m}$. See electronic annex in the online version of this article.

\section{CONCLUSIONS}

In this paper a methodology is presented that allows the inter-penetration areas associated with the undeformed geometries of the wheel and the rail surfaces to be located. The method is especially suitable for implementation in railway dynamics simulation models, which are based on the elastic contact hypothesis. The technique consists of the discretization of the surfaces in contact into a set of truncated cones (for the wheel) and points (for the rail). From this hypothesis, the areas of interpenetration are obtained through a closed-form expression. The robustness of the method is based on the fact that it is not possible to overlook any area of inter-penetration, which is a frequent problem in most of the methodologies that can be found in the literature.

The technique is applied to the calculation of the frictionless static position of the wheelset on the rails, when the lateral position and the yaw angle are given, and a vertical force is applied. To this end the CONTACT algorithm has been adopted as wheel-rail contact model. The results allow smooth functions to be calculated that determine the dependent coordinates of the wheelset as a function of the independent ones. The results show, for the profiles studied, positions in which the contact is approximately Hertzian, non-conformal and non-Hertzian, and conformal. 


\section{ACKNOWLEDGEMENTS}

The authors acknowledge the financial contribution of the European Union's Shift2Rail programme (RUN2Rail project), the Spanish Ministry of Economy, Industry and Competitiveness and the European Regional Development Fund (projects TRA2013-45596-C2-1-R and TRA2017-84701-R).

\section{REFERENCES}

[1] V.K. Garg, R.V. Dukkipati, Dynamics of railway vehicle systems, Academic Press, Toronto, 1984.

[2] A.H. Wickens, The dynamic stability of railway vehicle wheelsets and bogies having profiled wheels, International Journal of Solids and Structures, 1 (1965) 319-341.

[3] A.D. De Pater, The geometrical contact between track and wheelset, Vehicle System Dynamics, 17 (1988) 127-140.

[4] G. Yang, Dynamic analysis of railway wheelsets and complete vehicle systems, in: Laboratory for Engineering Mechanics, Delft University of Technology, Delft, 1993.

[5] D. Negretti, A third-order approximation method for three-dimensional wheel-rail contact, Vehicle System Dynamics, 50 (2012) 431-448.

[6] A.A. Shabana, K.E. Zaazaa, J.L. Escalona, J.R. Sany, Modeling two-point wheel/rail contacts using constraint and elastic-force approaches, in: American Society of Mechanical Engineers, Rail Transportation Division (Publication) RTD, 2002, pp. 35-50.

[7] H. Netter, G. Schupp, W. Rulka, K. Schroeder, New aspects of contact modelling and validation within multibody system simulation of railway vehicles, Vehicle System Dynamics, 29 (1998) 246-269.

[8] J. Pombo, J. Ambrósio, M. Silva, A new wheel-rail contact model for railway dynamics, Vehicle System Dynamics, 45 (2007) 165-189.

[9] O. Polach, Characteristic parameters of nonlinear wheel/rail contact geometry, Vehicle System Dynamics, 48 (2010) 19-36.

[10] J. Santamaría, E.G. Vadillo, J. Gómez, A comprehensive method for the elastic calculation of the two-point wheel-rail contact, Vehicle System Dynamics, 44 (2006) 240-250.

[11] J.L. Cuperus, G. Venter, Numerical simulation and parameterisation of rail-wheel normal contact, Proceedings of the Institution of Mechanical Engineers, Part F: Journal of Rail and Rapid Transit, 231 (2016) 419-430.

[12] H. Chollet, M. Sébès, J.L. Maupu, J.B. Ayasse, The VOCO multi-body software in the context of real-time simulation, Vehicle System Dynamics, 51 (2013) 570-580.

[13] J.-P. Pascal, B. Soua, Solving conformal contacts using multi-Hertzian techniques, Vehicle System Dynamics, 54 (2016) 784-813.

[14] J. Piotrowski, H. Chollet, Wheel-rail contact models for vehicle system dynamics including multipoint contact, Vehicle System Dynamics, 43 (2005) 455-483.

[15] E.A.H. Vollebregt, C. Weidemann, A. Kienberger, Use of "CONTACT" in multi-body vehicle dynamics and profile wear simulation: initial results, in: S. Iwinicki (Ed.) 22nd International Symposium on Dynamics of Vehicles on Roads and Tracks (IAVSD2011), Manchester, 2011.

[16] B.B. Liu, S. Bruni, E. Vollebregt, A non-Hertzian method for solving wheel-rail normal contact problem taking into account the effect of yaw, Vehicle System Dynamics, 54 (2016) 1226-1246.

[17] J.J. Kalker, Three-Dimensional Elastic Bodies in Rolling Contact, Springer Netherlands, Dordrecht, 1990.

[18] J. Pombo, J. Ambrosio, A computational efficient general wheel-rail contact detection method, Journal of Mechanical Science and Technology, 19 (2005) 411-421.

[19] I. Kaiser, K. Popp, Interaction of elastic wheelsets and elastic rails: modelling and simulation, Vehicle System Dynamics, 44 (2006) 932-939.

[20] S. Falomi, M. Malvezzi, E. Meli, Multibody modeling of railway vehicles: Innovative algorithms for the detection of wheel-rail contact points, Wear, 271 (2011) 453-461.

[21] E. Meli, S. Magheri, M. Malvezzi, Development and implementation of a differential elastic wheel-rail contact model for multibody applications, Vehicle System Dynamics, 49 (2011) 9691001. 
[22] K. Wang, The track of wheel contact points and the calculation of wheel/rail geometric contact parameters, Journal of Southwest Jiaotong University, 1 (1984) 89-99.

[23] N. Burgelman, The wheel-rail contact problem in vehicle dynamic simulation, in: Railahead Group, Technische Universiteit Delft, PhD Thesis, 2016.

[24] Z. Ren, S.D. Iwnicki, G. Xie, A new method for determining wheel-rail multi-point contact, Vehicle System Dynamics, 49 (2011) 1533-1551.

[25] X. Yang, S. Gu, S. Zhou, Y. Zhou, S. Lian, A method for improved accuracy in three dimensions for determining wheel/rail contact points, Vehicle System Dynamics, 53 (2015) 1620-1640.

[26] K.L. Johnson, Contact Mechanics, Cambridge University Press, Cambridge, 1985.

[27] European Standards, Railway applications - Testing for the acceptance of running characteristics of railway vehicles - Testing of running behaviour and stationary tests, in: EN 14363:2005.

\section{APPENDIX}

This appendix shows the formulation used to calculate parameters $\alpha$ and $\varepsilon$ from Equation (1). This development is only valid if the rotation matrix $\mathbf{A}$ satisfies $A_{31}=0$. First, the following variables have to be computed:

$$
\begin{gathered}
R=\left[\left(\bar{y}_{r}-y\right) A_{33}-\left(\bar{z}_{r}-z\right) A_{23}\right]\left(z_{w+1}-z_{w}\right)+\left(A_{23} A_{32}-A_{22} A_{33}\right)\left(y_{w} z_{w+1}-y_{w+1} z_{w}\right), \\
S=\left[\left(\bar{z}_{r}-z\right)\left(z_{w}-z_{w+1}\right)+\left(y_{w} z_{w+1}-y_{w+1} z_{w}\right) A_{32}\right] A_{21} \\
T=\left[\left(\bar{z}_{r}-z\right) A_{22}-\left(\bar{y}_{r}-y\right) A_{32}\right]\left(y_{w}-y_{w+1}\right)
\end{gathered}
$$

and

$$
d=R^{2}+S^{2}-T^{2}
$$

If $d<0$ then the line does not intersect the cone. Otherwise the parameters $\alpha$ and $\varepsilon$ are obtained from the following equations:

$$
\begin{gathered}
\varepsilon=2 \arctan \frac{S+\sqrt{d}}{R-T}, \\
\alpha=\frac{A_{32} y_{w}+A_{33} z_{w} \cos \varepsilon-\bar{z}_{r}+z}{\left(y_{w}-y_{w+1}\right) A_{32}+\left(z_{w}-z_{w+1}\right) A_{33} \cos \varepsilon} .
\end{gathered}
$$

Therefore the intersection conditions are satisfied if $d \geq 0$ and $\alpha$ is contained in the interval $] 0,1]$.

From Equations (1), (A.5) and (A.6), the longitudinal coordinate is obtained as follows:

$$
\bar{x}=\left(\alpha z_{w+1}+(1-\alpha) z_{w}\right)\left(A_{13} \cos \varepsilon-A_{11} \sin \varepsilon\right)+\left(\alpha y_{w+1}+(1-\alpha) y_{w}\right) A_{12} .
$$

